\title{
Mixed Monotone Iterative Technique for Impulsive Periodic Boundary Value Problems in Banach Spaces
}

\author{
Pengyu Chen \\ Department of Mathematics, Northwest Normal University, Lanzhou 730070, China \\ Correspondence should be addressed to Pengyu Chen, chpengyu123@163.com
}

Received 20 April 2010; Accepted 15 September 2010

Academic Editor: Feliz Manuel Minhós

Copyright (C) 2011 Pengyu Chen. This is an open access article distributed under the Creative Commons Attribution License, which permits unrestricted use, distribution, and reproduction in any medium, provided the original work is properly cited.

This paper deals with the existence of $L$-quasi-solutions for impulsive periodic boundary value problems in an ordered Banach space $E$. Under a new concept of upper and lower solutions, a new monotone iterative technique on periodic boundary value problems of impulsive differential equations has been established. Our result improves and extends some relevant results in abstract differential equations.

\section{Introduction}

The theory of impulsive differential equations is a new and important branch of differential equation theory, which has an extensive physical, chemical, biological, and engineering background and realistic mathematical model, and hence has been emerging as an important area of investigation in the last few decades; see [1]. Correspondingly, applications of the theory of impulsive differential equations to different areas were considered by many authors, and some basic results on impulsive differential equations have been obtained; see [2-5]. But many of them are about impulsive initial value problem; see $[2,3]$ and the references therein. The research on impulsive periodic boundary value problems is seldom; see $[4,5]$.

In this paper, we use a monotone iterative technique in the presence of coupled lower and upper $L$-quasisolutions to discuss the existence of solutions to the impulsive periodic boundary value problem (IPBVP) in an ordered Banach space $E$

$$
\begin{gathered}
u^{\prime}(t)=f(t, u(t), u(t)), \quad t \in J, \quad t \neq t_{k}, \\
\left.\Delta u\right|_{t=t_{k}}=I_{k}\left(u\left(t_{k}\right), u\left(t_{k}\right)\right), \quad k=1,2, \ldots, m, \\
u(0)=u(\omega),
\end{gathered}
$$


where $f \in C(J \times E \times E, E), J=[0, \omega], \omega>0 ; 0<t_{1}<t_{2}<\cdots<t_{m}<\omega ; I_{k} \in C(E \times E, E)$ is an impulsive function, $k=1,2, \ldots, m .\left.\Delta u\right|_{t=t_{k}}$ denotes the jump of $u(t)$ at $t=t_{k}$, that is, $\left.\Delta u\right|_{t=t_{k}}=u\left(t_{k}^{+}\right)-u\left(t_{k}^{-}\right)$, where $u\left(t_{k}^{+}\right)$and $u\left(t_{k}^{-}\right)$represent the right and left limits of $u(t)$ at $t=t_{k}$, respectively.

The monotone iterative technique in the presence of lower and upper solutions is an important method for seeking solutions of differential equations in abstract spaces. Early on, Lakshmikantham and Leela [4] built a monotone iterative method for the periodic boundary value problem of first-order differential equation in $\mathbb{R}$

$$
\begin{gathered}
u^{\prime}(t)=f(t, u(t)), \quad t \in J, \\
u(0)=u(\omega),
\end{gathered}
$$

and they proved that, if $\operatorname{PBVP}(1.2)$ has a lower solution $v_{0}$ and an upper solution $w_{0}$ with $v_{0} \leq w_{0}$, and nonlinear term $f$ satisfies the monoton condition

$$
f\left(t, x_{2}\right)-f\left(t, x_{1}\right) \geq-M\left(x_{2}-x_{1}\right), \quad \forall t \in J, v_{0}(t) \leq x_{1} \leq x_{2} \leq w_{0}(t),
$$

with a positive constant $M$, then $\operatorname{PBVP}(1.2)$ has minimal and maximal solutions between $v_{0}$ and $w_{0}$, which can be obtained by a monotone iterative procedure starting from $v_{0}$ and $w_{0}$, respectively. Later, $\mathrm{He}$ and $\mathrm{Yu}$ [5] developed the problem to impulsive differential equation

$$
\begin{gathered}
u^{\prime}(t)=f(t, u(t)), \quad t \in J, \quad t \neq t_{k}, \\
\left.\Delta u\right|_{t=t_{k}}=I_{k}\left(u\left(t_{k}\right)\right), \quad k=1,2, \ldots, m, \\
u(0)=u(\omega),
\end{gathered}
$$

where $f \in C(J \times \mathbb{R}, \mathbb{R}), J=[0, \omega], I_{k} \in C(\mathbb{R}, \mathbb{R}), 0<t_{1}<t_{2}<\cdots<t_{m}<\omega$.

But all of these results are in real spaces $\mathbb{R}$. We not only consider problems in Banach spaces, but also expand the nonlinear term to the case of $f(t, u, u)$. If $f(t, u)=f_{1}(t, u)+$ $f_{2}(t, u), f_{1}(t, u)$ is nondecreasing in $u$ and $f_{2}(t, u)$ is nonincreasing in $u$, then the monotonity condition (1.3) is not satisfied, and the results in $[4,5]$ are not right, in this case, we studied the IPBVP(1.1). As far as we know, no work has been done for the existence of solutions for $\operatorname{IPBVP}(1.1)$ in Banach spaces. impulse

In order to apply the monotone iterative technique to the initial value problem without

$$
\begin{gathered}
u^{\prime}(t)=f(t, u(t), u(t)), \quad t \in[0, a], \\
u(0)=x_{0},
\end{gathered}
$$

Lakshmikantham et al. [6] and Guo and Lakshmikantham [7] obtained the existence of coupled quasisolutions of problem (1.5) by mixed monotone sequence of coupled quasiupper and lower solutions under the concept of quasiupper and lower solutions. In this paper, we improve and extend the above-mentioned results, and obtain the existence of the coupled minimal and maximal $L$-quasisolutions and the solutions between the coupled minimal 
and maximal $L$-quasisolutions of the problem (1.1) through the mixed monotone iterative about the coupled $L$-quasisolutions. If $L \equiv 0$, the coupled upper and lower $L$-quasisolutions are equivalent to coupled upper and lower quasisolutions of the $\operatorname{IPBVP}(1.1)$. If $L \equiv 0$, $f(t, u, u)=f(t, u)$ and $I_{k}(u, u)=I_{k}(u)$, the coupled upper and lower L-quasisolutions are equivalent to upper and lower solutions of $\operatorname{IPBVP}(1.4)$.

\section{Preliminaries}

Let $E$ be an ordered Banach space with the norm $\|\cdot\|$ and partial order $\leq$, whose positive cone $P=\{x \in E \mid x \geq 0\}$ is normal with normal constant $N$. Let $J=[0, \omega], \omega>0$ is a constant; $0=t_{0}<t_{1}<t_{2}<\cdots<t_{m}<t_{m+1}=\omega ; J_{k}=\left[t_{k-1}, t_{k}\right], k=1,2, \ldots, m+1$, $J^{\prime}=J \backslash\left\{t_{1}, t_{2}, \ldots, t_{m}\right\}$. Let $\mathrm{PC}(J, E)=\left\{u: J \rightarrow E \mid u(t)\right.$ is continuous at $t \neq t_{k}$, and left continuous at $t=t_{k}$, and $u\left(t_{k}^{+}\right)$exists, $\left.k=1,2, \ldots, m\right\}$. Evidently, $\operatorname{PC}(J, E)$ is a Banach space with the norm $\|u\|_{\mathrm{PC}}=\sup _{t \in J}\|u(t)\|$. An abstract function $u \in \operatorname{PC}(J, E) \cap C^{1}\left(J^{\prime}, E\right)$ is called a solution of $\operatorname{IPBVP}(1.1)$ if $u(t)$ satisfies all the equalities of (1.1).

Let $\operatorname{PC}^{1}(J, E)=\left\{u \in \operatorname{PC}(J, E) \cap C^{1}\left(J^{\prime}, E\right) \mid u^{\prime}\left(t_{k}^{+}\right)\right.$and $u^{\prime}\left(t_{k}^{-}\right)$exist, $\left.k=1,2, \ldots, m\right\}$. For $u \in \mathrm{PC}^{1}(J, E)$, it is easy to see that the left derivative $u_{-}^{\prime}\left(t_{k}\right)$ of $u(t)$ at $t=t_{k}$ exists and $u_{-}^{\prime}\left(t_{k}\right)=u^{\prime}\left(t_{k}^{-}\right)$, and set $u^{\prime}\left(t_{k}\right)=u^{\prime}\left(t_{k}^{-}\right)$, then $u^{\prime} \in \operatorname{PC}(J, E)$. If $u \in \operatorname{PC}(J, E) \cap C^{1}\left(J^{\prime}, E\right)$ is a solution of $\operatorname{IPBVP}(1.1)$, by the continuity of $f, u \in \operatorname{PC}^{1}(J, E)$.

Let $C(J, E)$ denote the Banach space of all continuous $E$-value functions on interval $J$ with the norm $\|u\|_{C}=\max _{t \in J}\|u(t)\|$. Let $\alpha(\cdot)$ denote the Kuratowski measure of noncompactness of the bounded set. For the details of the definition and properties of the measure of noncompactness, see [8]. For any $B \subset C(J, E)$ and $t \in J$, set $B(t)=\{u(t) \mid u \in B\} \subset$ $E$. If $B$ is bounded in $C(J, E)$, then $B(t)$ is bounded in $E$, and $\alpha(B(t)) \leq \alpha(B)$.

Now, we first give the following lemmas in order to prove our main results.

Lemma 2.1 (see [9]). Let $B=\left\{u_{n}\right\} \subset \mathrm{PC}(J, E)$ be a bounded and countable set. Then $\alpha(B(t))$ is Lebesgue integral on $\mathrm{J}$, and

$$
\alpha\left(\left\{\int_{J} u_{n}(t) d t \mid n \in \mathbb{N}\right\}\right) \leq 2 \int_{J} \alpha(B(t)) d t .
$$

Lemma 2.2 (see [10]). Let $D \subset E$ be bounded. Then exist a countable set $D_{0} \subset D$, such that $\alpha(D) \leq 2 \alpha\left(D_{0}\right)$.

Lemma 2.3 (see [11]). Let $B \subset C(J, E)$ be equicontinuous. Then $\alpha(B(t))$ is continuous on $J$, and

$$
\alpha(B)=\max _{t \in J} \alpha(B(t))=\alpha(B(J))
$$

Lemma 2.4 (see [8]). Let $X$ be a Banach space and $\Omega$ is a bounded convex closed set in $X, Q: \Omega \rightarrow$ $\Omega$ be condensing, then $Q$ has a fixed point in $\Omega$. 
To prove our main results, for any $h \in \mathrm{PC}(J, E)$, we consider the periodic boundary value problem (PBVP) of linear impulsive differential equation in $E$

$$
\begin{gathered}
u^{\prime}(t)+M u(t)=h(t), \quad t \in J^{\prime}, \\
\left.\Delta u\right|_{t=t_{k}}=y_{k}, \quad k=1,2, \ldots, m, \\
u(0)=u(\omega),
\end{gathered}
$$

where $M \geq 0, y_{k} \in E, k=1,2, \ldots, m$.

Lemma 2.5. For any $h \in \mathrm{PC}(J, E), x \in E$ and $y_{k} \in E, k=1,2, \ldots, m$, the linear $P B V P(2.3)$ has a unique solution $u \in P C^{1}(J, E)$ given by

$$
u(t)=e^{-M t} y_{h}+\int_{0}^{t} e^{-M(t-s)} h(s) d s+\sum_{t_{k}<t} e^{-M\left(t-t_{k}\right)} y_{k}
$$

where $y_{h}=\left(1 /\left(1-e^{-M \omega}\right)\right)\left(\int_{0}^{\omega} e^{-M(\omega-s)} h(s) d s+\sum_{k=1}^{m} e^{-M\left(\omega-t_{k}\right)} y_{k}\right)$.

Proof. For any $h \in \mathrm{PC}(J, E), x \in E$ and $y_{k} \in E, k=1,2, \ldots, m$, the linear initial value problem

$$
\begin{gathered}
u^{\prime}(t)+M u(t)=h(t), \quad t \in J^{\prime}, \\
\left.\Delta u\right|_{t=t_{k}}=y_{k}, \quad k=1,2, \ldots, m, \\
u(0)=x
\end{gathered}
$$

has a unique solution $u \in \mathrm{PC}^{1}(J, E)$ given by

$$
u(t)=e^{-M t} x+\int_{0}^{t} e^{-M(t-s)} h(s) d s+\sum_{t_{k}<t} e^{-M\left(t-t_{k}\right)} y_{k}
$$

where $M \geq 0$ is a constant [3].

If $u$ is a solution of the linear initial value problem (2.5) satisfies $u(\omega)=x$, namely

$$
x=e^{-M \omega} x+\int_{0}^{\omega} e^{-M(\omega-s)} h(s) d s+\sum_{k=1}^{m} e^{-M\left(\omega-t_{k}\right)} y_{k}
$$

then it is the solution of the linear $\operatorname{PBVP}(2.3)$. From (2.7), we have

$$
x=\frac{1}{1-e^{-M \omega}}\left(\int_{0}^{\omega} e^{-M(\omega-s)} h(s) d s+\sum_{k=1}^{m} e^{-M\left(\omega-t_{k}\right)} y_{k}\right) \triangleq y_{h} .
$$

So, (2.4) is satisfied. 
Inversely, we can verify directly that the function $u \in P C(J, E)$ defined by (2.4) is a solution of the linear PBVP(2.3). Therefore, the conclusion of Lemma 2.5 holds.

Definition 2.6. Let $L \geq 0$ be a constant. If functions $v_{0}, w_{0} \in \operatorname{PC}^{1}(J, E)$ satisfy

$$
\begin{gathered}
v_{0}^{\prime}(t) \leq f\left(t, v_{0}(t), w_{0}(t)\right)+L\left(v_{0}(t)-w_{0}(t)\right), \quad t \in J^{\prime}, \\
\left.\Delta v_{0}\right|_{t=t_{k}} \leq I_{k}\left(v_{0}\left(t_{k}\right), w_{0}\left(t_{k}\right)\right), \quad k=1,2, \ldots, m, \\
v_{0}(0) \leq v_{0}(\omega), \\
w_{0}^{\prime}(t) \geq f\left(t, w_{0}(t), v_{0}(t)\right)+L\left(w_{0}(t)-v_{0}(t)\right), \quad t \in J^{\prime}, \\
\left.\Delta w_{0}\right|_{t=t_{k}} \geq I_{k}\left(w_{0}\left(t_{k}\right), v_{0}\left(t_{k}\right)\right), \quad k=1,2, \ldots, m, \\
w_{0}(0) \geq w_{0}(\omega),
\end{gathered}
$$

we call $v_{0}, w_{0}$ coupled lower and upper L-quasisolutions of the $\operatorname{IPBVP}(1.1)$. Only choose " $="$ in (2.9) and (2.10), we call $\left(v_{0}, w_{0}\right)$ coupled $L$-quasisolution pair of the IPBVP(1.1). Furthermore, if $v_{0}=w_{0}:=u_{0}$, we call $u_{0}$ a solution of the $\operatorname{IPBVP}(1.1)$.

Now, we define an operator $Q: \operatorname{PC}(J, E) \rightarrow \operatorname{PC}(J, E)$ as following:

$$
\begin{aligned}
Q(u, v)(t)= & e^{-M t} S(u, v)+\int_{0}^{t} e^{-M(t-s)}[f(s, u(s), v(s))+(M+L) u(s)-L v(s)] d s \\
& +\sum_{t_{k}<t} e^{-M\left(t-t_{k}\right)} I_{k}\left(u\left(t_{k}\right), v\left(t_{k}\right)\right)
\end{aligned}
$$

where

$$
\begin{gathered}
S(u, v)=\frac{1}{1-e^{-M \omega}}\left(\int_{0}^{\omega} e^{-M(\omega-s)}[f(s, u(s), v(s))+(M+L) u(s)-L v(s)] d s\right. \\
\left.+\sum_{k=1}^{m} e^{-M\left(\omega-t_{k}\right)} I_{k}\left(u\left(t_{k}\right), v\left(t_{k}\right)\right)\right) .
\end{gathered}
$$

Evidently, $\mathrm{PC}(J, E)$ is also an ordered Banach space with the partial order " $\leq$ " reduced by the positive cone $K_{\mathrm{PC}}=\{u \in \mathrm{PC}(J, E) \mid u(t) \geq 0, t \in J\}$. $K_{\mathrm{PC}}$ is also normal with the same normal constant $N$. For $v, w \in \operatorname{PC}(J, E)$ with $v \leq w$, we use $[v, w]$ to denote the order interval $\{u \in \operatorname{PC}(J, E) \mid v \leq u \leq w\}$ in $\operatorname{PC}(J, E)$, and $[v(t), w(t)]$ to denote the order interval $\{u \in E \mid v(t) \leq u(t) \leq w(t), t \in J\}$ in $E$. 


\section{Main Results}

Theorem 3.1. Let $E$ be an ordered Banach space, whose positive cone $P$ is normal, $f \in C(J \times E \times E, E)$ and $I_{k} \in C(E \times E, E), k=1,2, \ldots, m$. Assume that the IPBVP(1.1) has coupled lower and upper L-quasisolutions $v_{0}$ and $w_{0}$ with $v_{0} \leq w_{0}$. Suppose that the following conditions are satisfied:

(H1) There exist constants $M>0$ and $L \geq 0$ such that

$$
f\left(t, u_{2}, v_{2}\right)-f\left(t, u_{1}, v_{1}\right) \geq-M\left(u_{2}-u_{1}\right)-L\left(v_{1}-v_{2}\right),
$$

for any $t \in J$, and $v_{0}(t) \leq u_{1} \leq u_{2} \leq w_{0}(t), v_{0}(t) \leq v_{2} \leq v_{1} \leq w_{0}(t)$.

(H2) The impulsive function $I_{k}(\cdot, \cdot)$ satisfies

$$
I_{k}\left(u_{1}, v_{1}\right) \leq I_{k}\left(u_{2}, v_{2}\right), \quad k=1,2, \ldots, m,
$$

$$
\text { for any } t \in J \text {, and } v_{0}(t) \leq u_{1} \leq u_{2} \leq w_{0}(t), v_{0}(t) \leq v_{2} \leq v_{1} \leq w_{0}(t) .
$$

(H3) There exist a constant $L_{1}>0$ such that

$$
\alpha\left(\left\{f\left(t, u_{n}, v_{n}\right)+M u_{n}\right\}\right) \leq L_{1}\left(\alpha\left(\left\{u_{n}\right\}\right)+\alpha\left(\left\{v_{n}\right\}\right)\right),
$$

for all $t \in J$, and increasing or decreasing monotonic sequences $\left\{u_{n}\right\} \subset\left[v_{0}(t), w_{0}(t)\right]$ and $\left\{v_{n}\right\} \subset\left[v_{0}(t), w_{0}(t)\right]$.

(H4) The sequences $v_{n}(0)$ and $w_{n}(0)$ are convergent, where $v_{n}=Q\left(v_{n-1}, w_{n-1}\right), w_{n}=$ $Q\left(w_{n-1}, v_{n-1}\right), n=1,2, \ldots$

Then the IPBVP(1.1) has minimal and maximal coupled $L$-quasisolutions between $v_{0}$ and $w_{0}$, which can be obtained by a monotone iterative procedure starting from $v_{0}$ and $w_{0}$, respectively.

Proof. By the definition of $Q$ and Lemma 2.5, $Q:\left[v_{0}, w_{0}\right] \times\left[v_{0}, w_{0}\right] \rightarrow \operatorname{PC}(J, E)$ is continuous, and the coupled $L$-quasisolutions of the $\operatorname{IPBVP}(1.1)$ is equivalent to the coupled fixed point of operator $Q$. Combining this with the assumptions $(H 1)$ and $(H 2)$, we know $Q:\left[v_{0}, w_{0}\right] \times$ $\left[v_{0}, w_{0}\right] \rightarrow \mathrm{PC}(J, E)$ is a mixed monotone operator (about the mixed monotone operator, please see $[6,7])$.

Next, we show $v_{0} \leq Q\left(v_{0}, w_{0}\right), Q\left(w_{0}, v_{0}\right) \leq w_{0}$. Let $h(t)=v_{0}^{\prime}(t)+M v_{0}(t)$, by (2.9), $h \in \mathrm{PC}(J, E)$ and $h(t) \leq f\left(t, v_{0}, w_{0}\right)+(L+M) v_{0}-L w_{0}, t \in J^{\prime}$. By Lemma 2.5

$$
\begin{aligned}
v_{0}(t)= & \frac{e^{-M t}}{1-e^{-M \omega}}\left(\int_{0}^{\omega} e^{-M(\omega-s)} h(s) d s+\left.\sum_{k=1}^{m} e^{-M\left(\omega-t_{k}\right)} \Delta v_{0}\right|_{t=t_{k}}\right) \\
& +\int_{0}^{t} e^{-M(t-s)} h(s) d s+\left.\sum_{t_{k}<t} e^{-M\left(t-t_{k}\right)} \Delta v_{0}\right|_{t=t_{k}}
\end{aligned}
$$


Boundary Value Problems

$$
\begin{aligned}
\leq & e^{-M t} S\left(v_{0}, w_{0}\right)+\int_{0}^{t} e^{-M(t-s)}\left[f\left(s, v_{0}(s), w_{0}(s)\right)+(L+M) v_{0}(s)-L w_{0}(s)\right] d s \\
& +\sum_{t_{k}<t} e^{-M\left(t-t_{k}\right)} I_{k}\left(v_{0}\left(t_{k}\right), w_{0}\left(t_{k}\right)\right) \\
= & Q\left(v_{0}, w_{0}\right)(t), \quad t \in J,
\end{aligned}
$$

namely, $v_{0} \leq Q\left(v_{0}, w_{0}\right)$. Similarly, it can be show that $Q\left(w_{0}, v_{0}\right) \leq w_{0}$. So, $Q:\left[v_{0}, w_{0}\right] \times$ $\left[v_{0}, w_{0}\right] \rightarrow\left[v_{0}, w_{0}\right]$.

Now, we define two sequences $\left\{v_{n}\right\}$ and $\left\{w_{n}\right\}$ in $\left[v_{0}, w_{0}\right]$ by the iterative scheme

$$
v_{n}=Q\left(v_{n-1}, w_{n-1}\right), \quad w_{n}=Q\left(w_{n-1}, v_{n-1}\right), \quad n=1,2, \ldots
$$

Then from the mixed monotonicity of $Q$, it follows that

$$
v_{0} \leq v_{1} \leq v_{2} \leq \cdots \leq v_{n} \leq \cdots \leq w_{n} \leq \cdots \leq w_{2} \leq w_{1} \leq w_{0}
$$

We prove that $\left\{v_{n}\right\}$ and $\left\{w_{n}\right\}$ are uniformly convergent in $J$.

For convenience, let $B=\left\{v_{n} \mid n \in \mathbb{N}\right\}+\left\{w_{n} \mid n \in \mathbb{N}\right\}, B_{1}=\left\{v_{n} \mid n \in \mathbb{N}\right\}, B_{2}=$ $\left\{w_{n} \mid n \in \mathbb{N}\right\}, B_{10}=\left\{v_{n-1} \mid n \in \mathbb{N}\right\}$ and $B_{20}=\left\{w_{n-1} \mid n \in \mathbb{N}\right\}$. Since, $B_{1}=Q\left(B_{10}, B_{20}\right)$ and $B_{2}=Q\left(B_{20}, B_{10}\right)$, by (2.11) and the boundedness of $B_{10}$ and $B_{20}$, we easy see that $B_{1}$ and $B_{2}$ is equicontinuous in every interval $J_{k}^{\prime}$, so, $B$ is equicontinuous in every interval $J_{k^{\prime}}^{\prime}$, where $J_{1}^{\prime}=\left[0, t_{1}\right], J_{k}^{\prime}=\left(t_{k-1}, t_{k}\right], k=2,3, \ldots, m+1$. From $B_{10}=B_{1} \cup\left\{v_{0}\right\}$ and $B_{20}=B_{2} \cup\left\{w_{0}\right\}$ it follows that $\alpha\left(B_{10}(t)\right)=\alpha\left(B_{1}(t)\right)$ and $\alpha\left(B_{20}(t)\right)=\alpha\left(B_{2}(t)\right)$ for $t \in J$. Let $\varphi(t):=\alpha(B(t)), t \in J$, by Lemma 2.3, $\varphi \in \operatorname{PC}\left(J, \mathbb{R}^{+}\right)$. Going from $J_{1}^{\prime}$ to $J_{m+1}^{\prime}$ interval by interval we show that $\varphi(t) \equiv 0$ in $J$.

For $t \in J_{1}^{\prime}$, from (2.11), using Lemma 2.1 and assumption (H3) and (H4), we have

$$
\begin{aligned}
\varphi(t)=\alpha(B(t))=\alpha\left(B_{1}(t)+B_{2}(t)\right)=\alpha\left(Q\left(B_{10}, B_{20}\right)(t)+Q\left(B_{20}, B_{10}\right)(t)\right) \\
=\alpha\left(\left\{e^{-M t} S\left(v_{n-1}, w_{n-1}\right)\right.\right. \\
\quad+\int_{0}^{t} e^{-M(t-s)}\left[f\left(s, v_{n-1}(s), w_{n-1}(s)\right)+(L+M) v_{n-1}(s)-L w_{n-1}(s)\right] d s \\
\quad+e^{-M t} S\left(w_{n-1}, v_{n-1}\right) \\
\left.\left.\quad+\int_{0}^{t} e^{-M(t-s)}\left[f\left(s, w_{n-1}(s), v_{n-1}(s)\right)+(L+M) w_{n-1}(s)-L v_{n-1}(s)\right] d s\right\}\right)
\end{aligned}
$$




$$
\begin{aligned}
& \leq \alpha\left(\left\{e^{-M t} v_{n}(0)\right\}\right)+\alpha\left(\left\{e^{-M t} w_{n}(0)\right\}\right) \\
& \quad+2 \int_{0}^{t} e^{-M(t-s)} \alpha\left(\left\{f\left(s, v_{n-1}(s), w_{n-1}(s)\right)+(L+M) v_{n-1}(s)-L w_{n-1}(s)\right.\right. \\
& \left.\left.\quad+f\left(s, w_{n-1}(s), v_{n-1}(s)\right)+(L+M) w_{n-1}(s)-L v_{n-1}(s)\right\}\right) d s \\
& \leq 2 \int_{0}^{t} 2 L_{1}\left(\alpha\left(B_{10}(s)\right)+\alpha\left(B_{20}(s)\right)\right) d s \\
& \leq 8 L_{1} \int_{0}^{t} \varphi(s) d s .
\end{aligned}
$$

Hence by the Belman inequality, $\varphi(t) \equiv 0$ in $J_{1}^{\prime}$. In particular, $\alpha\left(B_{10}\left(t_{1}\right)\right)=0, \alpha\left(B_{20}\left(t_{1}\right)\right)=0$, this means that $B_{10}\left(t_{1}\right)$ and $B_{20}\left(t_{1}\right)$ are precompact in $E$. Thus $I_{1}\left(B_{10}\left(t_{1}\right)\right)$ and $I_{1}\left(B_{20}\left(t_{1}\right)\right)$ are precompact in $E$, and $\alpha\left(I_{1}\left(B_{10}\left(t_{1}\right)\right)\right)=0, \alpha\left(I_{1}\left(B_{20}\left(t_{1}\right)\right)\right)=0$.

Now, for $t \in J_{2}^{\prime}$, by (2.11) and the above argument for $t \in J_{1}^{\prime}$, we have

$$
\begin{aligned}
\varphi(t)= & \alpha(B(t))=\alpha\left(B_{1}(t)+B_{2}(t)\right)=\alpha\left(Q\left(B_{10}, B_{20}\right)(t)+Q\left(B_{20}, B_{10}\right)(t)\right) \\
\leq \alpha & \left\{e^{-M t} S\left(v_{n-1}, w_{n-1}\right)\right. \\
& \quad+\int_{0}^{t} e^{-M(t-s)}\left[f\left(s, v_{n-1}(s), w_{n-1}(s)\right)+(L+M) v_{n-1}(s)-L w_{n-1}(s)\right] d s \\
& +e^{-M t} S\left(w_{n-1}, v_{n-1}\right) \\
& \left.\left.+\int_{0}^{t} e^{-M(t-s)}\left[f\left(s, w_{n-1}(s), v_{n-1}(s)\right)+(L+M) w_{n-1}(s)-L v_{n-1}(s)\right] d s\right\}\right) \\
& +\alpha\left(I_{1}\left(B_{10}\left(t_{1}\right)\right)\right)+\alpha\left(I_{1}\left(B_{20}\left(t_{1}\right)\right)\right) \\
\leq & 8 L_{1} \int_{0}^{t} \varphi(s) d s=8 L_{1} \int_{t_{1}}^{t} \varphi(s) d s .
\end{aligned}
$$

Again by Belman inequality, $\varphi(t) \equiv 0$ in $J_{2}^{\prime}$, from which we obtain that $\alpha\left(B_{10}\left(t_{2}\right)\right)=0$, $\alpha\left(B_{20}\left(t_{2}\right)\right)=0$ and $\alpha\left(I_{2}\left(B_{10}\left(t_{2}\right)\right)\right)=0, \alpha\left(I_{2}\left(B_{20}\left(t_{2}\right)\right)\right)=0$.

Continuing such a process interval by intervai up to $J_{m+1}^{\prime}$, we can prove that $\varphi(t) \equiv 0$ in every $J_{k}^{\prime}, k=1,2, \ldots, m+1$.

For any $J_{k}$, if we modify the value of $v_{n}, w_{n}$ at $t=t_{k-1}$ via $v_{n}\left(t_{k-1}\right)=v_{n}\left(t_{k-1}^{+}\right)$, $w_{n}\left(t_{k-1}\right)=w_{n}\left(t_{k-1}^{+}\right), n \in \mathbb{N}$, then $\left\{v_{n}\right\}+\left\{w_{n}\right\} \subset C\left(J_{k}, E\right)$ and it is equicontinuous. Since $\alpha\left(\left\{v_{n}(t)\right\}+\left\{w_{n}(t)\right\}\right)=0,\left\{v_{n}(t)\right\}+\left\{w_{n}(t)\right\}$ is precompact in $E$ for every $t \in J_{k}$. By the ArzelaAscoli theorem, $\left\{v_{n}\right\}+\left\{w_{n}\right\}$ is precompact in $C\left(J_{k}, E\right)$. Hence, $\left\{v_{n}\right\}+\left\{w_{n}\right\}$ has a convergent subsequence in $C\left(J_{k}, E\right)$. Combining this with the monotonicity (3.6), we easily prove that $\left\{v_{n}\right\}+\left\{w_{n}\right\}$ itself is convergent in $C\left(J_{k}, E\right)$. In particular, $\left\{v_{n}(t)\right\}+\left\{w_{n}(t)\right\}$ is uniformly 
convergent over the whole of $J$. Hence, $\left\{v_{n}\right\}+\left\{w_{n}\right\}$ is uniformly convergent in $\operatorname{PC}(J, E)$. Set

$$
\underline{u}=\lim _{n \rightarrow \infty} v_{n}, \quad \bar{u}=\lim _{n \rightarrow \infty} w_{n}, \quad \text { in } \operatorname{PC}(J, E) .
$$

Letting $n \rightarrow \infty$ in (3.5) and (3.6), we see that $v_{0} \leq \underline{u} \leq \bar{u} \leq w_{0}$ and $\underline{u}=Q(\underline{u}, \bar{u}), \bar{u}=Q(\bar{u}, \underline{u})$. By the mixed monotonicity of $Q$, it is easy to see that $\underline{u}$ and $\bar{u}$ are the minimal and maximal coupled fixed points of $Q$ in $\left[v_{0}, w_{0}\right]$, and therefore, they are the minimal and maximal coupled $L$-quasisolutions of the $\operatorname{IPBVP}(1.1)$ in $\left[v_{0}, w_{0}\right]$, respectively.

In Theorem 3.1, if $E$ is weakly sequentially complete, condition (H3) and (H4) hold automatically. In fact, by Theorem 2.2 in [12], any monotonic and order-bounded sequence is precompact. By the monotonicity (3.6) and the same method in proof of Theorem 3.1, we can easily see that $\left\{v_{n}(t)\right\}$ and $\left\{w_{n}(t)\right\}$ are convergent on $J$. In particular, $\left\{v_{n}(0)\right\}$ and $\left\{w_{n}(0)\right\}$ are convergent. So, condition $(H 4)$ holds. Let $\left\{u_{n}\right\}$ and $\left\{v_{n}\right\}$ be increasing or decreasing sequences obeying condition (H3), then by condition (H1), $\left\{f\left(t, u_{n}, v_{n}\right)+M u_{n}\right\}$ is a monotonic and order-bounded sequence, so $\alpha\left(\left\{f\left(t, u_{n}, v_{n}\right)+M u_{n}\right\}\right)=0$. Hence, condition (H3) holds. From Theorem 3.1, we obtain the following corollary.

Corollary 3.2. Let $E$ be an ordered and weakly sequentially complete Banach space, whose positive cone $P$ is normal, $f \in C(J \times E \times E, E)$ and $I_{k} \in C(E \times E, E), k=1,2, \ldots, m$. If the IPBVP(1.1) has coupled lower and upper L-quasisolutions $v_{0}$ and $w_{0}$ with $v_{0} \leq w_{0}$, and conditions (H1) and (H2) are satisfied, then the IPBVP(1.1) has minimal and maximal coupled L-quasisolutions between $v_{0}$ and $w_{0}$, which can be obtained by a monotone iterative procedure starting from $v_{0}$ and $w_{0}$ respectively.

If we replace the assumption $(H 3)$ by the following assumption:

(H5) There exist positive constants $\bar{M}$ and $\bar{L}$ such that

$$
f\left(t, u_{2}, v_{2}\right)-f\left(t, u_{1}, v_{1}\right) \leq \bar{M}\left(u_{2}-u_{1}\right)+\bar{L}\left(v_{1}-v_{2}\right),
$$

$$
\text { for any } t \in J \text {, and } v_{0}(t) \leq u_{1} \leq u_{2} \leq w_{0}(t), v_{0}(t) \leq v_{2} \leq v_{1} \leq w_{0}(t) .
$$

We have the following result.

Theorem 3.3. Let $E$ be an ordered Banach space, whose positive cone $P$ is normal, $f \in C(J \times E \times E, E)$ and $I_{k} \in C(E \times E, E), k=1,2, \ldots, m$. If the IPBVP(1.1) has coupled lower and upper L-quasisolutions $v_{0}$ and $w_{0}$ with $v_{0} \leq w_{0}$, and conditions (H1), (H2), (H4) and (H5) hold, then the IPBVP(1.1) has minimal and maximal coupled L-quasisolutions between $v_{0}$ and $w_{0}$, which can be obtained by a monotone iterative procedure starting from $v_{0}$ and $w_{0}$ respectively.

Proof. For $t \in J$, let $\left\{u_{n}\right\} \subset\left[v_{0}(t), w_{0}(t)\right]$ be a increasing sequence and $\left\{v_{n}\right\} \subset\left[v_{0}(t), w_{0}(t)\right]$ be a decreasing sequence. For $m, n \in \mathbb{N}$ with $m>n$, by (H1) and (H5),

$$
\begin{aligned}
\theta & \leq f\left(t, u_{m}, v_{m}\right)-f\left(t, u_{n}, v_{n}\right)+M\left(u_{m}-u_{n}\right)+L\left(v_{n}-v_{m}\right) \\
& \leq(M+\bar{M})\left(u_{m}-u_{n}\right)+(L+\bar{L})\left(v_{n}-v_{m}\right) .
\end{aligned}
$$


By this and the normality of cone $P$, we have

$$
\begin{aligned}
& \left\|f\left(t, u_{m}, v_{m}\right)-f\left(t, u_{n}, v_{n}\right)+M\left(u_{m}-u_{n}\right)\right\| \\
& \quad \leq N\left\|(M+\bar{M})\left(u_{m}-u_{n}\right)+(L+\bar{L})\left(v_{n}-v_{m}\right)\right\|+L\left\|v_{n}-v_{m}\right\| \\
& \quad \leq N(M+\bar{M})\left\|u_{m}-u_{n}\right\|+(N(L+\bar{L})+L)\left\|v_{n}-v_{m}\right\| .
\end{aligned}
$$

From this inequality and the definition of the measure noncompactness, it follows that

$$
\begin{aligned}
\alpha\left(\left\{f\left(t, u_{n}, v_{n}\right)+M u_{n}\right\}\right) & \leq N(M+\bar{M}) \alpha\left(\left\{u_{n}\right\}\right)+(N(L+\bar{L})+L) \alpha\left(\left\{v_{n}\right\}\right) \\
& \leq L_{1}\left(\alpha\left(\left\{u_{n}\right\}\right)+\alpha\left(\left\{v_{n}\right\}\right)\right)
\end{aligned}
$$

where $L_{1}=N(M+\bar{M})+N(L+\bar{L})+L$. If $\left\{u_{n}\right\}$ is a increasing sequence and $\left\{v_{n}\right\}$ is a decreasing sequence, the above inequality is also valid. Hence $(H 3)$ holds.

Therefore, by Theorem 3.1, the IPBVP(1.1) has minimal and maximal coupled $L$ quasisolutions between $v_{0}$ and $w_{0}$, which can be obtained by a monotone iterative procedure starting from $v_{0}$ and $w_{0}$, respectively.

Now, we discuss the existence of the solution to the IPBVP(1.1) between the minimal and maximal coupled $L$-quasisolutions $\underline{u}$ and $\bar{u}$. If we replace the assumptions (H2) and (H3) by the following assumptions:

$(H 2)^{*}$ The impulsive function $I_{k}(\cdot, \cdot)$ satisfies

$$
I_{k}\left(u_{1}, v_{1}\right) \leq I_{k}\left(u_{2}, v_{2}\right), \quad k=1,2, \ldots, m,
$$

for any $t \in J$, and $v_{0}(t) \leq u_{1} \leq u_{2} \leq w_{0}(t), v_{0}(t) \leq v_{2} \leq v_{1} \leq w_{0}(t)$; and there exist $M_{k}>0, \sum_{k=1}^{m} M_{k} \leq\left(\left(8 L_{1} \omega-1\right)+\left(1-16 L_{1} \omega\right) e^{M \omega}\right) / 2\left(2 e^{M \omega}-1\right)$, such that

$$
\alpha\left(I_{k}\left(\left\{u_{n}\left(t_{k}\right)\right\} \times\left\{v_{n}\left(t_{k}\right)\right\}\right)\right) \leq M_{k}\left[\alpha\left(u_{n}\left(t_{k}\right)\right)+\alpha\left(v_{n}\left(t_{k}\right)\right)\right],
$$

for any countable sets $\left\{u_{n}\right\}$ and $\left\{v_{n}\right\}$ in $\left[v_{0}(t), w_{0}(t)\right]$.

$(H 3)^{*}$ There exist a constant $L_{1}>0$ such that

$$
\alpha\left(f\left(t, D_{1} \times D_{2}\right)+M D_{1}\right) \leq L_{1}\left(\alpha\left(D_{1}\right)+\alpha\left(D_{2}\right)\right),
$$

for any $t \in J$, where $D_{1}=\left\{v_{n}\right\}$ and $D_{2}=\left\{w_{n}\right\}$ are countable sets in $\left[v_{0}(t), w_{0}(t)\right]$.

We have the following existence result.

Theorem 3.4. Let $E$ be an ordered Banach space, whose positive cone $P$ is normal, $f \in C(J \times E \times E, E)$ and $I_{k} \in C(E \times E, E), k=1,2, \ldots, m$. If the IPBVP(1.1) has coupled lower and upper L-quasisolutions $v_{0}$ and $w_{0}$ with $v_{0} \leq w_{0}$, such that assumptions $(\mathrm{H} 1),(\mathrm{H} 2)^{*},(\mathrm{H} 3)^{*}$ and $(\mathrm{H} 4)$ hold, then the $\operatorname{IPBVP}(1.1)$ has minimal and maximal coupled L-quasisolutions $\underline{u}$ and $\bar{u}$ between $v_{0}$ and $w_{0}$, and at least has one solution between $\underline{u}$ and $\bar{u}$. 
Proof. We can easily see that $(\mathrm{H} 2)^{*} \Rightarrow(\mathrm{H} 2),(\mathrm{H} 3)^{*} \Rightarrow(H 3)$. Hence, by the Theorem 3.1 , the $\operatorname{IPBVP}(1.1)$ has minimal and maximal coupled $L$-quasisolutions $\underline{u}$ and $\bar{u}$ between $v_{0}$ and $w_{0}$. Next, we prove the existence of the solution of the equation between $\underline{u}$ and $\bar{u}$. Let $A u=$ $Q(u, u)$, clearly, $A:\left[v_{0}, w_{0}\right] \rightarrow\left[v_{0}, w_{0}\right]$ is continuous and the solution of the $\operatorname{IPBVP}(1.1)$ is equivalent to the fixed point of operator $A$. Since $A(D)$ is bounded and equicontinuous for any $D \subset\left[v_{0}, w_{0}\right]$, by Lemma 2.2, there exist a countable set $D_{0}=\left\{u_{n}\right\}$, such that

$$
\alpha(A(D)) \leq 2 \alpha\left(A\left(D_{0}\right)\right)
$$

By assumptions $(\mathrm{H} 2)^{*}$ and $(H 3)^{*}$ and Lemma 2.1,

$$
\begin{aligned}
& \alpha\left(A\left(D_{0}(t)\right)\right) \\
& =\alpha\left(\left\{\frac { e ^ { - M \omega } } { 1 - e ^ { - M \omega } } \left[\int_{0}^{\omega} e^{-M(t-s)}\left(f\left(s, u_{n}(s), u_{n}(s)\right)+M u_{n}(s)\right) d s\right.\right.\right. \\
& \left.\quad+\sum_{k=1}^{m} e^{-M\left(t-t_{k}\right)} I_{k}\left(u_{n}\left(t_{k}\right), u_{n}\left(t_{k}\right)\right)\right] \\
& \quad+\int_{0}^{t} e^{-M(t-s)}\left(f\left(s, u_{n}(s), u_{n}(s)\right)+M u_{n}(s)\right) d s \\
& \left.\left.\quad+\sum_{t_{k}<t} e^{-M\left(t-t_{k}\right)} I_{k}\left(u_{n}\left(t_{k}\right), u_{n}\left(t_{k}\right)\right)\right\}\right) \\
& \leq \frac{1}{e^{M \omega}-1}\left[2 \int_{0}^{\omega} e^{-M(t-s)} \alpha\left(f\left(s, D_{0}(s), D_{0}(s)\right)+M D_{0}(s)\right) d s\right. \\
& \left.\quad+\sum_{k=1}^{m} e^{-M\left(t-t_{k}\right)} \alpha\left(I_{k}\left(D_{0}\left(t_{k}\right), D_{0}\left(t_{k}\right)\right)\right)\right] \\
& \quad+2 \int_{0}^{t} e^{-M(t-s)} \alpha\left(f\left(s, D_{0}(s), D_{0}(s)\right)+M D_{0}(s)\right) d s+\sum_{t_{k}<t} e^{-M\left(t-t_{k}\right)} \alpha\left(I_{k}\left(D_{0}\left(t_{k}\right), D_{0}\left(t_{k}\right)\right)\right) \\
& <\frac{e^{M \omega}}{e^{M \omega}-1}\left[4 L_{1} \int_{0}^{\omega} \alpha\left(D_{0}(s)\right) d s+2 \sum_{k=1}^{m} M_{k} \alpha\left(D_{0}\left(t_{k}\right)\right)\right] \\
& \quad+4 L_{1} \int_{0}^{t} \alpha\left(D_{0}(s)\right) d s+2 \sum_{t_{k}<t} M_{k} \alpha\left(D_{0}\left(t_{k}\right)\right) \\
& \leq \frac{2 e^{M \omega}-1}{e^{M \omega}-1}\left(4 L_{1} \omega+2 \sum_{k=1}^{m} M_{k}\right) \alpha(D) .
\end{aligned}
$$

Since $A\left(D_{0}\right)$ is equicontinuous, by Lemma 2.3, $\alpha\left(A\left(D_{0}\right)\right)=\max _{t \in J} \alpha\left(A\left(D_{0}\right)(t)\right)$. Combing (3.17) and $(H 2)^{*}$. 
We have

$$
\alpha(A(D))<\frac{4 e^{M \omega}-2}{e^{M \omega}-1}\left(4 L_{1} \omega+2 \sum_{k=1}^{m} M_{k}\right) \alpha(D) \leq \alpha(D) .
$$

Hence, the operator $A:\left[v_{0}, w_{0}\right] \rightarrow\left[v_{0}, w_{0}\right]$ is condensing, by the Lemma 2.4, $A$ has fixed point $u$ in $\left[v_{0}, w_{0}\right]$.

Lastly, since $u=A u=Q(u, u), v_{0} \leq u \leq w_{0}$, by the mixed monotonity of $Q$

$$
v_{1}=Q\left(v_{0}, w_{0}\right) \leq Q(u, u) \leq Q\left(w_{0}, v_{0}\right)=w_{1}
$$

Similarly, $v_{2} \leq u \leq w_{2}$, in general, $v_{n} \leq u \leq w_{n}$, letting $n \rightarrow \infty$, we get $\underline{u} \leq u \leq \bar{u}$. Therefore, the IPBVP(1.1) at least has one solution between $\underline{u}$ and $\bar{u}$.

Remark 3.5. If $f(t, u, u)=f(t, u)$ and $I_{k}(u, u)=I_{k}(u)$, then Theorems 3.1, 3.3 and 3.4 are generalizations of the main results of [5] in Banach spaces.

Remark 3.6. If $f(t, u, u)=f(t, u)$ and $I_{k} \equiv 0$, then Theorems 3.1, 3.3 and 3.4 are generalizations of the Theory 4.1 of [4] in Banach spaces.

\section{An Example}

Consider the PBVP of infinite system for nonlinear impulsive differential equations:

$$
\begin{gathered}
u_{n}^{\prime}(t)=u_{n}(t)+\frac{1}{u_{n+2}(t)}, \quad 0 \leq t \leq \pi, t \neq \frac{\pi}{2} \\
\left.\Delta u_{n}\right|_{t=\pi / 2}=3 u_{n}\left(\frac{\pi}{2}\right)-2 u_{n+1}\left(\frac{\pi}{2}\right) \\
u_{n}(0)=u_{n}(\omega) \quad(n=1,2, \ldots) .
\end{gathered}
$$

\subsection{Conclusion}

$\operatorname{IPBVP}(4.1)$ has minimal and maximal coupled $L$-quasisolutions.

Proof. Let $\omega=\pi, E=l^{2}=\left\{u=\left.\left(u_{1}, \ldots, u_{n}, \ldots\right)\left|\sum_{n=1}^{\infty}\right| u_{n}\right|^{2}<\infty\right\}$ with norm $\|u\|=$ $\left(\sum_{n=1}^{\infty}\left|u_{n}\right|^{2}\right)^{1 / 2}$ and $P=\left\{u=\left(u_{1}, \ldots, u_{n}, \ldots\right) \in l^{2} \mid u_{n} \geq 0, n=1,2, \ldots\right\}$. Then $E$ is a weakly sequentially complete Banach space and $P$ is normal cone in $E$. IPBVP (4.1) can be regarded as an PBVP of the form (1.1) in $E$. In this case, $J=[0, \pi], v=\left(v_{1}, \ldots, v_{n}, \ldots\right), w=\left(w_{1}, \ldots, w_{n}, \ldots\right)$ and $f=\left(f_{1}, \ldots, f_{n}, \ldots\right)$, in which

$$
f_{n}(t, u, v)=u_{n}(t)+\frac{1}{v_{n+2}(t)}, \quad n=1,2, \ldots
$$

$k=1, t_{1}=\pi / 2$ and $I_{1 n}(u, v)=3 u_{n}-2 v_{n+1}, n=1,2, \ldots$ 
Evidently, $f \in C(J \times E \times E, E), I_{1} \in C(E \times E, E)$. Let

$$
\begin{gathered}
v_{0}(t)= \begin{cases}\left(-\cos t, \ldots,-\frac{\cos t}{n}, \ldots\right), & 0 \leq t \leq \frac{\pi}{2}, \\
\left(-1-\cos t, \ldots,-\frac{1+\cos t}{n}, \ldots\right), & \frac{\pi}{2}<t \leq \pi\end{cases} \\
w_{0}(t)= \begin{cases}\left(\cos t, \ldots, \frac{\cos t}{n}, \ldots\right), & 0 \leq t \leq \frac{\pi}{2} \\
\left(1+\cos t, \ldots, \frac{1+\cos t}{n}, \ldots\right), & \frac{\pi}{2}<t \leq \pi\end{cases}
\end{gathered}
$$

$L=1 / 2, M=1$. Then it is easy to verify that $v_{0}, w_{0}$ are coupled lower and upper $1 / 2$ quasisolutions of the $\operatorname{IPBVP}(4.1)$, and conditions $\left(H_{1}\right),\left(H_{2}\right)$ hold. Hence, our conclusion follows from Corollary 3.2.

\section{Acknowledgments}

This paper was supported by NNSF of China (10871160), the NSF of Gansu Province (0710RJZA103), and Project of NWNU-KJCXGC-3-47.

\section{References}

[1] V. Lakshmikantham, D. D. Bănov, and P. S. Simeonov, Theory of Impulsive Differential Equations, vol. 6 of Series in Modern Applied Mathematics, World Scientific, Teaneck, NJ, USA, 1989.

[2] D. J. Guo and X. Liu, "Extremal solutions of nonlinear impulsive integrodifferential equations in Banach spaces," Journal of Mathematical Analysis and Applications, vol. 177, no. 2, pp. 538-552, 1993.

[3] Y. Li and Z. Liu, "Monotone iterative technique for addressing impulsive integro-differential equations in Banach spaces," Nonlinear Analysis: Theory, Methods E Applications, vol. 66, no. 1, pp. 83-92, 2007.

[4] V. Lakshmikantham and S. Leela, "Existence and monotone method for periodic solutions of firstorder differential equations," Journal of Mathematical Analysis and Applications, vol. 91, no. 1, pp. 237$243,1983$.

[5] Z. He and J. Yu, "Periodic boundary value problem for first-order impulsive ordinary differential equations," Journal of Mathematical Analysis and Applications, vol. 272, no. 1, pp. 67-78, 2002.

[6] V. Lakshmikantham, S. Leela, and A. S. Vatsala, "Method of quasi-upper and lower solutions in abstract cones," Nonlinear Analysis, vol. 6, no. 8, pp. 833-838, 1982.

[7] D. J. Guo and V. Lakshmikantham, "Coupled fixed points of nonlinear operators with applications," Nonlinear Analysis: Theory, Methods \& Applications, vol. 11, no. 5, pp. 623-632, 1987.

[8] K. Deimling, Nonlinear Functional Analysis, Springer, Berlin, Germany, 1985.

[9] H.-P. Heinz, "On the behaviour of measures of noncompactness with respect to differentiation and integration of vector-valued functions," Nonlinear Analysis: Theory, Methods \& Applications, vol. 7, no. 12, pp. 1351-1371, 1983.

[10] Y. X. Li, "Existence of solutions to initial value problems for abstract semilinear evolution equations," Acta Mathematica Sinica, vol. 48, no. 6, pp. 1089-1094, 2005 (Chinese).

[11] D. J. Guo and J. X. Sun, Ordinary Differential Equations in Abstract Spaces, Shandong Science and Technology, Jinan, China, 1989.

[12] Y. H. Du, "Fixed points of increasing operators in ordered Banach spaces and applications," Applicable Analysis, vol. 38, no. 1-2, pp. 1-20, 1990. 